American Journal of Applied Sciences 3 (7): 1924-1928, 2006

ISSN 1546-9239

(c) 2006 Science Publications

\title{
Development of a Prototype Automated Sorting System for Plastic Recycling
}

\author{
D. A. Wahab, A. Hussain, E. Scavino, M.M. Mustafa and H. Basri \\ Faculty of Engineering, Universiti Kebangsaan Malaysia, 43600 UKM Bangi, Selangor DE, Malaysia
}

\begin{abstract}
Automated sorting for plastic recyclables has been seen as the way forward in the plastic recycling industry. Automated sorting provides significant improvements in terms of efficiency and consistency in the sorting process. In the case of macro sorting, which is the most common type of automated sorting, efficiency is determined by the mechanical details of the material handling system as well as the detection system. This paper provides a review on the state of-the-art technologies that have been deployed by some of the recycling facilities abroad. The design and development of a cost effective prototype automated system for sorting plastic recyclables is proposed and discussed.
\end{abstract}

Key words: Automated sorting, image processing, material handling, plastic recyclables, recycling

\section{INTRODUCTION}

Plastic materials are generally light, able to contain most consumable and household liquids and as such, are one of the highly utilized materials for domestic packaging. Plastic packaging can be made of different types of resins and the most common are PET, HDPE, LDPE, PVC, PP and PS as listed in Table 1. They are among the type of plastic materials that have numerous recycled applications. There are two methods for sorting recyclables; either through manual sorting or using automated systems. For plastic materials, sorting is normally based on the resin categories. This is important due to the fact that for plastic materials to be recycled into useable resins, a pure stream of resin must be obtained. Inefficient sorting that leads to a combination of different types of resins for example, PVC and PET can result in the release of hydrochloric gases $^{[1]}$.

Manual sorting relies on plant personnel who visually identify and pick plastic bottles as they travel along the conveyor belt ${ }^{[2]}$. These bottles are then sorted into the respective containers. Manual sorting may not be a suitable option for recycling facilities of high throughput. It has also been noted that the high turnover among sorting line workers had caused difficulties in achieving consistency in the plastic separation process $^{[3]}$.

Automated sorting systems employ a detection system or a combination of detection systems to identify the different types of plastic recyclable. These detection systems utilize state-of-the art technologies to automatically sort plastics either by resin type, shape, color or both. Automated sorting for plastic material can be either whole bottle sorting (macro-sorting) or flake sorting (micro-sorting). Flake sorting systems sort plastic in granulated form and are able to handle large volume of the material ${ }^{[2]}$.

\begin{tabular}{llll} 
Table 1: & Plastic bottle recycle code system and material \\
\hline Code & Material & $\begin{array}{l}\text { Code } \\
\text { System }\end{array}$ & Material \\
system & & 1-PETE & 5-PP \\
& Polyethylene & \\
Terephthalate
\end{tabular}

State-of-the-art technologies in automated sorting: Whilst flake-sorting system or microsorting is gaining acceptance in the recycling industry, whole bottle sorting or macrosorting is still the most prevalent form of automated sorting. During macrosorting, a detection system will detect the plastic recyclables based on its resin type or colour or even its physical characteristics. Upon analysis, these plastic bottles will be removed from the conveyor belt into their respective storage bin, using ejectors such as high-pressure air jets ${ }^{[4]}$. Optical Sorting systems, Near Infrared Systems and X-Ray technologies are some of the common technologies used in automated sorting systems.

Optical Sorting Systems use visible light to separate HDPE plastic bottles by color. Optical systems use black and white cameras for single colour separation, for example, detecting coloured bottles in a stream of non-pigmented, natural HDPE bottles. Optical sorting systems that provide multiple color separations use a colour camera. Commercially available systems can now sort a wide variety of colours including very faint translucent colored bottles. 
Near Infrared (NIR) Systems is a portion of the light spectrum that is invisible to the human eye. When plastic bottles are exposed to NIR signals, each plastic resin absorbs specific wavelengths and transmits others that constitute unique characteristic responses, which are then detected by sensors. NIR can be designed to differentiate between a wide range of plastic resin types. The advantage of using NIR is that surface contaminants such as labels do not affect the efficiency of the sorting process. However, it is important to note that NIR detection systems cannot detect bottles that are blocked by another packaging. It is therefore critical for bottles to be presented to the detector one at a time to maximize accuracy and therefore quality. X-rays are used for identifying PVC plastics as it detects the presence of the chlorine atom found in PVC bottles from its unique peak in the X-ray spectrum. The use of $\mathrm{X}$-Ray technology however, would require registration and compliance to the relevant OSHA regulations ${ }^{[2]}$.

The development of systems to automatically sort post consumer plastic has started since 1989 and two years later the first commercial system that separates PVC from PET using X-Ray technology, was introduced $^{[5]}$. Since then, research and development efforts in this area has escalated rapidly, more so with the increasing awareness on engineering life cycle issues such as design for recycling, reuse, remanufacture etc.

The current automated systems provide efficient sorting for plastic materials. However, it requires high investment in the specific technologies and machineries. The use of these technologies are however inappropriate for low to medium recycling volume for example in countries such as Malaysia. In view of that, a study has been conducted to determine the viability of imaging technology for automated sorting. For purposes of this initial study, plastic recyclables of PET/PETE, HDPE and PVC resin type were identified and classified into two main classes of plastic recyclables: PET and non PET with the HDPE and PVC belong to the non PET class. The PET bottles are mainly designed for edible liquid such as mineral water and drinking juice while the non PET bottles are commonly used for chemical based liquid such as household detergent, pesticide etc.

System description: The intelligent sorting system comprised the material handling system and the detection system. In a sorting process for either whole bottle or flake sorting, proper feeding and preparation of the feed stream is critical to the optimal separation efficiency of the recyclables and at least equally important as the quality of the detection system used ${ }^{[5]}$. The main components of the material handling system are the hopper, conveyor belt, the ejector and the collecting bins for the different types of recyclables while the detection system is made up of the machine vision system. Figure 1 illustrates the main components of the automated sorting system.

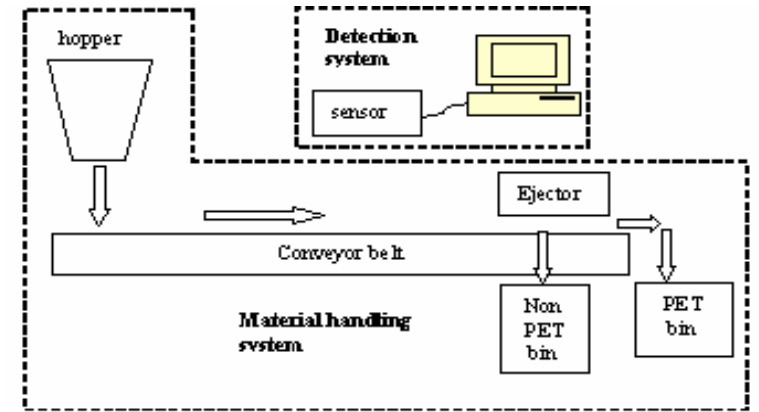

Fig. 1: A diagrammatic illustration of the automated sorting system

For the purpose of the prototype system, recyclables shall be dropped onto the hopper and allowed to fall in a horizontal position and single-filed as they move along the conveyor belt. This requirement is similar to an automated system that uses NIR as the detector, whereby recyclables shall be presented to the detector one at a time to maximize accuracy and therefore quality. The prototype system shall be able to handle, detect and sort plastic recyclables of various sizes from as small as a Vitagen ${ }^{\circledR}$ bottle $(150 \mathrm{ml})$ to a recyclable as large as a domestic cooking oil plastic container $(1500 \mathrm{ml})$. For the purpose of the prototype, the category of plastic materials that can be separated is limited to PET and non-PET materials only.

The material handling system comprised the hopper and the conveyor system. The design of each system component underwent the design methodology process namely identification of design requirements, generation of design concepts and evaluation of the concepts in view of selecting the final design concept.

In defining the requirements for the hopper and the conveyor system, constraints such as the length of the conveyor belt, capabilities of the detector and cost were taken into consideration.

The design requirements for the material handling system were specified as follows:

i. Capable of holding a small batch of plastic recyclables of various shapes and sizes, from as small as a Vitagen ${ }^{\circledR}$ bottle to a $1.5 l$ cooking oil container;

ii. Capable of controlling the movement and flow rate of the plastic recyclables in the hopper. The flow shall be smooth in order to avoid congestion at the exit of the hopper.

iii. Capable of dropping plastic recyclables one at a time on to the conveyor belt so as to prevent overlapping of the recyclables as they move along the conveyor belt. This is to facilitate detection and separation of the different types of plastic recyclables.

Development of the system structure for the machine vision module: During the initial stage, the viability of machine vision for detecting plastic material 
was studied. Initially, sorting was based on the material used for the plastic bottles. By limiting the types of plastics to be sorted to PET/PETE and non-PET materials, the focus of the study would only be on 2categorical pattern recognition task. The outcome of this research can be integrated as an embedded framework to develop the automated system using machine vision. Figure 2 shows the overall system design structure, which consists of three main components namely, the pre-processing, feature extraction and classification. For the development of the automated sorting system, image-processing techniques were used. It involved the feature extraction and image recognition modules i.e. the sorting of plastic bottles/packages was based on images. The images were preprocessed and several features such as the histogram, area, perimeter, centroid, hue etc. were extracted beforehand.

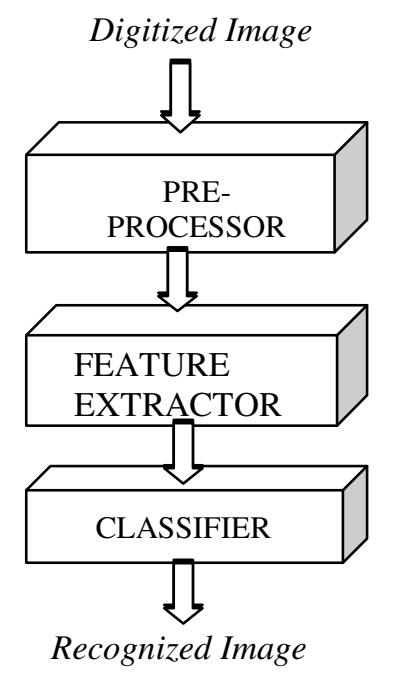

Fig. 2: The overall system structure

The Pre-processing component performs the following operations: thresholding, morphological operation, grid to acquire uniform image and edge detection via boundary tracing algorithm. Thresholding is a non-linear operation that converts a gray-scale image into a binary image where the two levels are assigned to pixels that are below or above the specified threshold value.

Morphological operation applies a structuring element to an input image, creating an output image of the same size. The most basic morphological operations are dilation and erosion. Edge detection refers to the process of identifying and locating sharp discontinuities in an image.

The preprocessed images are further analyzed to extract important features to represent the images in a compact and reduced form. In this work, feature extractions were based on the shape and color context of the plastic bottles.

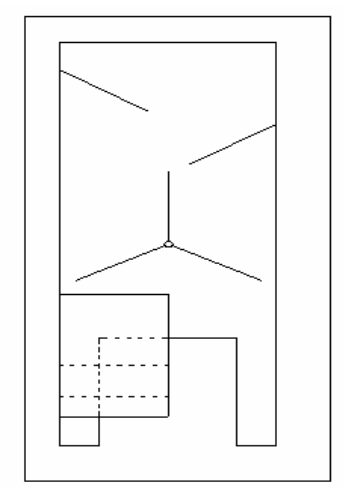

Fig. 3: An example of the design concept (front view) for the hopper

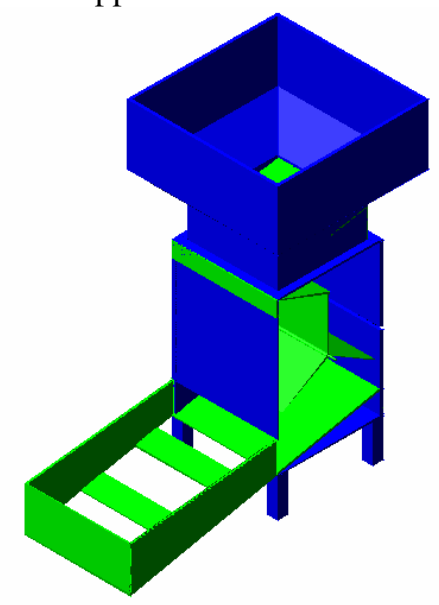

Fig. 4: A rendered image of the final design concept

There are three main approaches to shape representation: 1) boundary or contour-based 2) region or area based and 3) skeleton based ${ }^{[6]}$. The identified feature vectors are histogram of the images, histogram of FCC, Fourier descriptor and centroidal method. Thus far, the boundary-based approach was implemented using Freeman Chain Code (FCC) algorithm, which was introduced by Herbert Freeman in 1961.

\section{Design and development work}

Design of the hopper: Challenges in designing the hopper lies in the requirement for handling recyclables of different shapes and sizes whilst ensuring a singulated flow on the conveyor belt.

Several design concepts were generated and evaluated based on the design requirements. Figure 3 shows an example of the design concept generated for the hopper. The hopper was designed with two plates attached to the wall. These plates were placed at different heights and on opposite sides of the wall. The function of the plates is to reduce the flowrate of the recyclables. At the bottom of the hopper is a rotating blade that gently rotates for the same purpose. Based on this concept, several other design options were generated and proposed including the use of two rotating blades; one at the upper part and another at the 
lower part of the hopper. The upper rotating blade rotates at a slower speed compared to the lower rotating blade in order to control the flowrate of the recyclables. All the proposed concepts were evaluated and a final concept was selected as shown in Fig. 4. The final concept incorporates a special purpose tray consisting of different apertures sizes based on the size categories of the recyclables. The tray which acts as a flow control mechanism is placed at a declining position at the exit of the hopper.

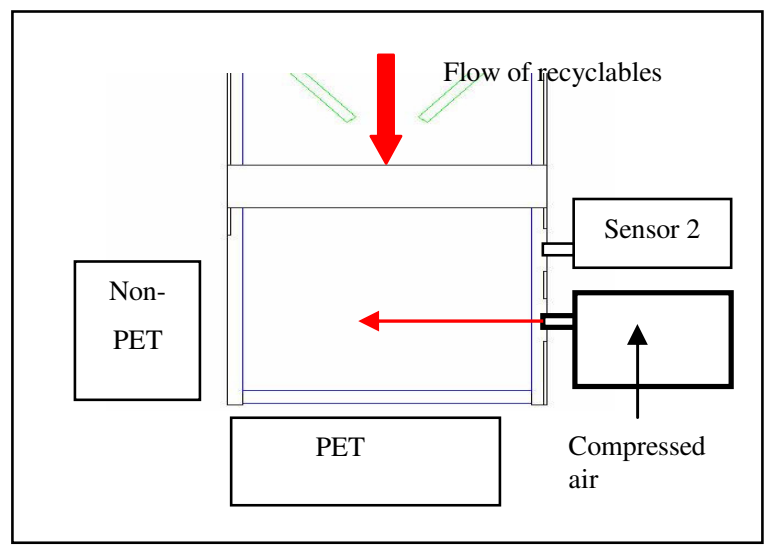

Fig. 5: An example of the ejector using compressed air (top view)

For the purpose of the prototype, the length of the conveyor was limited to $1.5 \mathrm{~m}$. An ejector that separates the non-PET from the PET material shall be placed at the end of the conveyor belt. Several ejecting mechanisms were proposed including the use of a movable plate that flaps down $45^{\circ}$ or $90^{\circ}$ from its horizontal position depending on the type of materials for the respective bins. Other options include the use of a pressurised air jet (Fig. 5) and a double acting pneumatic cylinder. Upon detection by the vision system, these ejectors will eject non-PET from the material flow, into their respective collecting bins.

Each design concept was evaluated based on the specified requirements. The use of pressurized air jets was felt to be inappropriate for use in the prototype system due to difficulties in controlling the amount and direction of the air from the orifice. The pressure of the air jet required to blow off a sample of higher density for example, a shampoo bottle made of High Density Polyethylene (HDPE) material would certainly differ from a much lighter recyclable for example, a Vitagen ${ }^{\circledR}$ bottle. For the prototype system, pneumatic cylinder was selected as the ejector.

The main requirement for the machine vision system is the capability to identify plastic bottles arriving at random times in random positions and orientations on a moving conveyor belt. Moreover, the speed in the image acquisition and treatment as well as in pattern recognition plays a fundamental role for industrial applications in view of the amount of waste to be processed.

When considering the economics of a machine vision system for the identification and sorting of

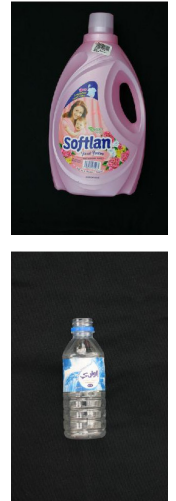

(a)

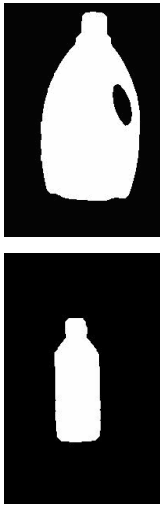

(b)

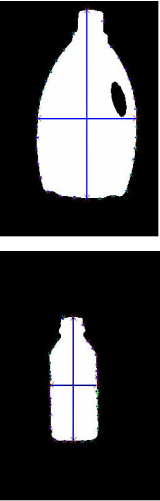

(c)
Fig. 6: Non PET and PET sample for (a) original (b) binarized and (c) feature

plastic bottles, the most cost effective choice was for a cheap commercially available computer camera for image acquisition. A customize software was developed using standard image processing techniques; feature extraction and classification were performed by analysis of shape as well as artificial intelligence techniques.

The camera, a commercially available color webcam, was placed vertically over a stretch of the conveyor belt in order to encompass its whole width. The repetition rate of the camera alone was limited by the amount of light on the object plane and was typically $1 / 20$ s in laboratory conditions. At present, the camera is capable of taking a new image every $1 / 5$ s, i.e. after analysis of the previously acquired image. The identification of objects in an image was performed by calculating the gradient of each color plane. An edge point was selected if the sum of the three gradients was over a pre-selected threshold. Both, the raw image and the binary image obtained using the edge detection was treated with standard noise reduction filters.

The extraction of features was based on the shape of the binarized image. This technique is fast and reliable and allows the identification of multiple objects in a single image; however it requires a database containing all the features of known bottles and it is not capable of identifying a new specimen when it appears on the conveyor belt. Nevertheless, even in an industrial application, all unidentified objects can be easily separated for insertion in the database and then recirculated for processing.

The database on features of known bottles was built for testing purposes. It consists of a set of 35 different plastic bottles and contains a set of features for every bottle including the type of plastic. The extracted features include the length and width along the principal axes, the area, the aspect ratio, and the perimeter evaluated in polar coordinates out of the area centroid of the binarized image over 90 predefined angles. The color properties of the original image were not taken into account at this stage because of the extreme influence of the lighting conditions.

Several techniques have been tested for the pattern matching and the identification of the plastic bottles in 
an image. The Euclidean distance between a subset of stored and measured features proved to be very reliable even in cases of bottles with similar shape. Several artificial neural network models were considered ${ }^{[7,8]}$ and the Self Organized Maps (SOM, or Kohonen maps) was used in this implementation in which it learns the geometrical dimensions in pixels of the image. Moreover, a group of neural networks were trained to identify the objects with the perimeter in polar coordinates as input. The output of each pattern recognition technique was a "factor of merit" whose value indicated the likelihood of positive identification. The final choice could be simply the highest value of all factors of merit or a combination.

As the aim of this prototype was a selective repartition of PET and non PET objects, all the bottles which were not positively identified as PET would trigger an extraction routine which activated the pneumatic cylinder.

\section{RESULTS AND DISCUSSION}

This prototype system was tested for speed and accuracy. The requirement for rapid industrial processes has led to the deployment of shape analysis method for the identification of meaningful objects. In this study, a non-compiled software has been developed on a standard PC. In this configuration, it is possible to perform the whole procedure from image acquisition to classification in $\sim 150 \mathrm{~ms}$ regardless of the number of objects in the image. The system alone would be capable of processing a minimum of 400,000 bottles in a day based on a 24-hour operating scheme while requiring very little human intervention, if any.

The sorting accuracy was evaluated for each pattern recognition technique, under normal laboratory lighting. Every bottle of the database was dropped on the conveyor belt dozens of times under varying lighting condition. When considering the accuracy of the final classification for each kind of bottle, all techniques provided around $95 \%$ of correct identifications, with smaller values around $80 \%$ in cases of poor lighting conditions. In such cases, failure in the procedure would generally be due to the lack of edge identification rather than during the pattern recognition phase. At this stage, mistakes in identification were mostly due to confusion on very similar $0.5 l$ water bottles, posing similar challenges to a sorting operator. When considering the final classification in terms of the material type, accuracy reaches almost $100 \%$. However, under poor lighting condition some mismatch may occur.

\section{CONCLUSION}

Existing automated sorting systems that deploy state-of-the-art technology such as Near Infra-Red, Infra Red and X-ray require high investments and are justifiable for recycling facilities of high throughput. In Malaysia, despite the growing awareness on recycling, the activity is still at its infancy. The recycling volume at the local Plastic Recycling Facilities (PRF) can be categorized as low to medium, hence the need for a cheaper yet efficient technology. This paper has described the development of a prototype automated sorting system comprising the material handling system and the material detection system using image processing. With the use of machine intelligence, a hazard-free mechanism for sorting plastic materials was developed. With further improvement in its capability and efficiency, the proposed system would be capable of providing a much cleaner and more cost effective method for plastic recycling.

Further studies will be conducted in view of improving both the speed and the accuracy of the system. Speed can be increased by building a standalone electronic module in order to avoid the sharing of resources of a normal $\mathrm{PC}$, hence a reduction in costs and material. Accuracy can be improved by controlling the lighting of the objects and setting up a hood in order to avoid varying light from the surrounding areas. In such controlled conditions, new refined routines could involve the use of color and brightness as a further tool for pattern recognition. A refinement of the routines, involving artificial intelligence techniques also in the edge detection could be useful for high-speed conveyor belts which would tend to blur the acquired images.

\section{ACKNOWLEDGEMENTS}

The authors would like to thank UKM for sponsoring this work under Fundamental Project KK012/2004.

\section{REFERENCES}

1. Bruno, E.A., Automated Sorting of Plastics for Recycling. http://www.p2pays.org.

2. Clean Washington Center, Automated Sorting Systems for Bottles and Regrind in Sorting HDPE Bottles From Other Plastic and Non-Plastic Containers: Introduction and Overview. http://www.cwc.org/hdpe/hdpe4.htm pp: 9-18.

3. Dinger, P., 1997. Automated sorting for mixed plastics, Citing Internet sources URL

4. http://www.cepis.ops.oms.org/muwww/full. May 2004

5. Phillips, M., 2001. Sorting it all out , Citing Internet sources, URL http://www.recyclingtoday.com. 29 Jan. 2004.

6. Kenny, G.R., M.G. Roe and Hottenstein. Automated Plastic Bottle Sorting Systems.

7. Economic and Technical Experience and New Resin Identification, R'99. http://www.magsep.com/PprR99.htm, pp: 1-10.

8. Gonzalez, R. C. and R.E. Woods, 2002. Digital Image processing. Upper Saddle River. Prentice Hall. 